\title{
A mixing timescale model for TPDF simulations of turbulent premixed flames
}

\author{
Michael Kuron ${ }^{a^{*}}$, Zhuyin Ren ${ }^{b^{*}}$, Evatt R. Hawkes ${ }^{c}$, Hua Zhou ${ }^{b}$, \\ Hemanth Kolla ${ }^{d}$, Jacqueline H. Chen ${ }^{d}$, Tianfeng $\mathrm{Lu}^{a}$ \\ ${ }^{a}$ Department of Mechanical Engineering, University of Connecticut, Storrs, CT 06269, USA \\ ${ }^{b}$ Center for Combustion Energy and School of Aerospace Engineering, Tsinghua University, \\ Beijing 100084, China \\ ${ }^{c}$ School of Photovoltaic and Renewable Energy Engineering, The University of New South \\ Wales, Sydney, NSW 2052, Australia \\ ${ }^{d}$ Combustion Research Facility, Sandia National Laboratories, Livermore, CA 96551, USA \\ *Corresponding Author Email: michael.kuron@uconn.edu, zhuyinren@tsinghua.edu.cn
}

\begin{abstract}
Transported probability density function (TPDF) methods are an attractive modeling approach for turbulent flames as chemical reactions appear in closed form. However, molecular micro-mixing needs to be modeled and this modeling is considered a primary challenge for TPDF methods. In the present study, a new algebraic mixing rate model for TPDF simulations of turbulent premixed flames is proposed, which is a key ingredient in commonly used molecular mixing models. The new model aims to properly account for the transition in reactive scalar mixing rate behavior from the limit of turbulence-dominated mixing to molecular mixing behavior in flamelets. An a priori assessment of the new model is performed using direct numerical simulation (DNS) data of a lean premixed hydrogen-air jet flame. The new model accurately captures the mixing timescale behavior in the DNS and is found to be a significant improvement over the commonly used constant mechanical-to-scalar mixing timescale ratio model. An a posteriori TPDF study is then performed using the same DNS data as a numerical test bed. The DNS provides the initial conditions and time-varying input quantities, including the mean velocity, turbulent diffusion coefficient, and modeled scalar mixing rate for the TPDF simulations, thus allowing an exclusive focus on the mixing model. The new mixing timescale model is compared with the constant mechanical-to-scalar mixing timescale ratio coupled with the Euclidean Minimum Spanning Tree (EMST) mixing model, as well as a laminar flamelet closure by Pope and Anand (S.B. Pope, M.S. Anand, Proc. Combust. Inst. 20 (1984) 403-410). It is found that the laminar flamelet closure is unable to properly capture the mixing behavior in the thin reaction zones regime while the constant mechanical-to-scalar mixing timescale model under-predicts the flame speed. The EMST model coupled with the new mixing timescale model provides the best prediction of the flame structure and flame propagation among the models tested, as the dynamics of reactive scalar mixing across different flame regimes are appropriately accounted for.
\end{abstract}

Keywords: Transported Probability Density Function, Turbulent Premixed Flames, Euclidean Minimum Spanning Tree, Mixing Timescale Models, Direct Numerical Simulation 


\section{Introduction}

The prediction of the mean chemical reaction rate in turbulent premixed flames in both Reynolds-averaged Navier-Stokes (RANS) and large-eddy simulations (LES) simulations remains a challenging problem in turbulent combustion modelling [1]. Transported probability density function (TPDF) methods provide an elegant solution to the closure of the mean chemical source term in turbulent flames and have been extensively studied in the context of turbulent reacting flows [1-6]. Despite some reported success, e.g. in [7,8], challenges remain when applying the TPDF method to turbulent premixed flames, as the complex flame dynamics and turbulence-chemistry interactions make it difficult to develop predictive models across different flame regimes. In particular, while the chemical reaction term is treated exactly, the molecular mixing term is unclosed in the TPDF method. Premixed flames are arguably much more challenging than non-premixed flames because local gradients of controlling scalars, e.g. a progress variable, are driven principally by reaction-diffusion balances, rather than by the straining-diffusion balances that determine gradients of the main controlling scalar in nonpremixed combustion, i.e. mixture fraction. This is made evident by a recent study of the TPDF method in which simple mixing closures were deemed sufficient for strongly burning nonpremixed flames [9].

Modeling of the molecular mixing term is considered to be a primary challenge in the TPDF methods [10-12]. Lindstedt and Vaos [13] found that the mixing models have a crucial impact on the computed turbulent burning velocities and that some mixing models suffer from significant shortcomings. More recently, Rowinski and Pope studied a series of piloted premixed jet flames [14] with strong finite-rate chemistry effects using the joint velocity-turbulence frequency-composition PDF method $[10,11]$. They found that the reaction progress is over- 
predicted to varying degrees depending on the jet velocity and that the mixing model behavior in this combustion regime is the likely cause for the observed discrepancy in reaction progress. As in previous PDF calculations of the premixed flames $[7,8]$, the primary aspect of the mixing models called into question is the specification of the mechanical-to-scalar timescale ratio, which is assumed to be constant, regardless of the combustion regime.

Many approaches to the closure of the molecular mixing term have been developed in the literature, and mixing models such as the interaction by exchange with the mean (IEM) [15] or Linear Mean-Square Estimation model [16], modified Curl (MC) [17-19], and Euclidean minimum spanning tree (EMST) $[20,21]$ have been widely adopted in the TPDF methods due to the simplicity in implementation and guarantee of realizability. Approaches such as the binomial Langevin model [22] and the mapping closure mixing model $[23,24]$ have demonstrated excellent performance in simple test cases, however may suffer from an implementation dependence on the species ordering as noted in [25]. Other stochastic mixing model approaches include the Fokker-Planck closure models of [26], which require the specification of the joint conditional scalar dissipation rate, a challenging task in its own right. A comprehensive appraisal of different mixing models for PDF simulations of turbulent premixed flames itself is an important topic but lies outside of the scope of this work.

While these mixing models describe the manner in which mixing occurs, each requires the specification of a Favre-averaged mixing rate, which is defined based on a controlling scalar $\phi$ as $\Omega=\widetilde{\chi_{\phi}} / \widetilde{\phi^{\prime \prime 2}}$ where $\widetilde{\chi_{\phi}}=2 D_{\phi} \nabla \widetilde{\phi " \nabla \phi}$ is the Favre-averaged scalar dissipation rate, $\widetilde{\phi^{\prime \prime 2}}$ is the Favre-averaged scalar variance [2], and $D_{\phi}$ is the scalar's diffusivity. A recent a posteriori study of molecular mixing models using direct numerical simulation (DNS) data demonstrated that both mixing model formulation and the scalar mixing rate are crucial for turbulent premixed 
combustion, and that the EMST mixing model is capable of accurately modeling the micromixing in a turbulent premixed flame provided that an accurate model of the mixing rate can be provided [27].

In typical TPDF simulations [11,28], the scalar mixing rate is assumed to be linearly proportional to the turbulent mixing rate. This approach is founded on studies of passive scalar mixing, and does not adequately describe the mixing dynamics of reactive scalars in the limit of large Damköhler number [29]. Several algebraic models for the mixing rate of reactive scalars that account for the effects of small scale turbulent mixing as well as reaction and dilatation have been proposed [30-32]. However, these models do not properly recover the correct mixing behavior in the limit of thin flamelets [33]. Closures specific to flamelet-regime turbulent premixed flames, such as that of Pope and Anand [34], close the mixing and reaction term by mapping the mixture state to a 1D freely propagating premixed flame, have also been proposed. However, the intrinsic assumption of embedded laminar flamelets limits the applicability of such closures, as they are unable to capture the transition to more intense turbulent combustion regimes. Another issue is that this fast-chemistry, premixed-flame specific approach throws away the significant advantages of PDF methods in treating problems involving relatively slow processes such as NOx or soot formation.

In the present work, a new algebraic mixing rate model is proposed that aims to model the transition in scalar mixing rate behavior from the limit of turbulence-dominated mixing to thin flamelet mixing behavior. A variant of this hybrid timescale model has been proposed and preliminarily studied for PDF simulations of turbulent premixed flames [35], though systematic a priori and a posteriori testing is not reported. In the remainder of this paper, we first derive the mixing rate model in section 2 and then perform an a priori comparison of the new model 
with a previously reported series of DNS modelling temporally evolving premixed hydrogen-air slot jet flames [36]. Finally, an a posteriori TPDF evaluation of the new mixing rate model is undertaken by using DNS as a numerical test bed for the TPDF solution.

\section{Timescale Model Development}

As described in [33], reactive scalar mixing rates in turbulent premixed flames depend on the local state of both the flow turbulence and the chemical reactions, which can be characterized by the Damköhler number, $D a=\tau_{\text {turb }} / \tau_{\text {chem }}$, where $\tau_{\text {turb }}$ and $\tau_{\text {chem }}$ are characteristic turbulence and chemical timescales, respectively. In the limit of large global Damköhler number, reactions occur in propagating thin structures that resemble laminar flames and thus scalar mixing rates can be strongly affected by the chemical timescales. Conversely, for low global Damköhler number, the scalar mixing rate can be expected to be controlled by the small scale turbulent motions, which in turn are driven by large-scale motions, as is found to be a reasonable approximation in studies of passive scalar mixing [1]. Kolla et al. demonstrated by an order of magnitude analysis that for finite values of the Damköhler number, the exact transport equation for the Favre-averaged scalar dissipation rate of the progress variable, $\widetilde{\chi_{c}}$, can be approximated to leading order by the effects of dilatation, turbulence-scalar interaction, chemical reaction, and molecular dissipation as in Equation 1 [31].

$$
2 \overline{\rho \chi_{c} \frac{\partial u_{l}}{\partial x_{l}}}-2 \overline{\rho \Gamma_{c}}\left(\frac{\partial c^{\prime \prime}}{\partial x_{j}} \frac{\widehat{\partial u^{\prime \prime}}}{\partial x_{k}} \frac{\partial c^{\prime \prime}}{\partial x_{k}}\right)+2\left(\overline{\frac{\partial c^{\prime \prime}}{\partial x_{k}} \frac{\partial \dot{\omega}^{\prime \prime}}{\partial x_{k}}}\right)-\overline{2 \rho\left(\Gamma_{c} \frac{\partial^{2} c^{\prime \prime}}{\partial x_{j} \partial x_{k}}\right)^{2}} \approx 0
$$

The terms in Equation 1 require closure, and the model proposed by Kolla et al. to account for the dominant physics of reactive scalar mixing can be written as:

$$
\widetilde{\chi_{C}}=\frac{C_{3} \tilde{\varepsilon}}{\beta^{\prime}} \widetilde{\tilde{k}} c^{\widetilde{2} 2}+\frac{1}{\beta^{\prime}}\left(2 K_{c}^{*} \frac{S_{L}^{0}}{\delta_{L}^{0}}-\tau C_{4} D a_{L}\right) \widetilde{c^{\prime \prime 2}}
$$


where $C_{3}, C_{4}$, and $\beta^{\prime}$ are model constants; $K_{c}^{*}$ is a constant computed based on the laminar flame structure; $\tau=\left(T_{a d}-T_{u}\right) / T_{u}$ is the heat release parameter with $T_{a d}$ and $T_{u}$ defined as the adiabatic flame temperature and the unburnt mixture temperature, respectively; and $D a_{L}=\frac{s_{L}^{0} / \delta_{L}^{0}}{\tilde{\varepsilon} / \tilde{k}}$ with $S_{L}^{0}$ and $\delta_{L}^{0}$ representing the unstrained laminar flame speed and thermal thickness, respectively. The first term in this equation accounts for the influence of turbulence on the scalar mixing, while the second term is to account for the influence of the flame structure. However, as noted by Bray et al. [33], this model and others of its kind do not properly recover the correct mixing rate in the flamelet limit, which merits further investigation with DNS data.

A new mixing timescale model is hereby proposed to account for both flamelet-controlled and turbulence-controlled mixing to more accurately model local mixing rates. In the limit of passive scalar mixing, Mantel and Borghi [37] showed by an order of magnitude analysis of the Favre-averaged scalar dissipation rate transport equation that the turbulence-scalar interaction and molecular dissipation terms in Equation 1 are balanced in the leading order. They proposed a model for the mixing rate that is proportional to the turbulence timescale according to the classical expression in Equation 3.

$$
\Omega_{t}=\widetilde{\chi_{\phi}} / \widetilde{\phi^{\prime 2}}=C_{\phi} \tau_{\text {turb }}{ }^{-1}
$$

The constant of proportionality $C_{\phi}$ is the mechanical-to-scalar timescale ratio and is typically taken to be 2.0 based on studies of passive scalar mixing in shear flows [38], which in order of magnitude is also supported by DNS of reactive non-premixed jet flames [39]. In the RANS context, the turbulence timescale is defined as the ratio of the turbulent kinetic energy, $\tilde{k}$, to the turbulent kinetic energy dissipation rate, $\tilde{\varepsilon}$.

Conversely, in the limit of laminar flamelets embedded in a turbulent flow field, the mixing rate is expected to be dependent on the laminar flame structure. Assuming that the premixed 
flamelets can be adequately described by a single reaction progress variable, c, the Favreaveraged mixing rate can be expressed in terms of the conditional scalar dissipation rate, $\left\langle\chi_{c} \mid c\right\rangle$, as

$$
\Omega_{f}=\widetilde{\chi_{c}} / \widetilde{c^{\prime \prime 2}}=\int_{0}^{1}\left\langle\chi_{c} \mid \zeta\right\rangle \widetilde{P}_{c}(\zeta) d \zeta / \widetilde{c^{\prime \prime 2}}
$$

where $\widetilde{P}_{c}(\zeta)$ is the Favre-averaged probability density function of the progress variable and $\zeta$ is the sample space variable. This expression is advantageous in that the PDF of the progress variable is computed naturally as a part of the TPDF solution, and thus only a model for the conditional scalar dissipation rate in the flamelet limit is required. As a first attempt, we close the model by reconstructing the conditional scalar dissipation rate from a $1 \mathrm{D}$ freely propagating premixed flame. The model format implies that the large variation in the mixing rate can be primarily attributed to the strong local variation in the progress variable PDF and that the local flamelets are reasonably approximated by the conditional scalar dissipation rate of the freely propagating flame at a known unburnt condition. Note that the model can in principle account for the strain/stretch effect on the flame structure by reconstructing the conditional scalar dissipation rate from a stretched premixed flame.

In practice, local mixing conditions in a turbulent premixed flame may vary such that neither of these limiting assumptions are valid for the entire flow field, and thus some intermediate local state between the two limits must be sought. To accomplish this, we define the segregation factor, $\eta=\widetilde{c^{12}} /[\tilde{c}(1-\tilde{c})],[33,40]$ to act as the blending variable between the two limiting states. As the local Damköhler number tends to infinity and the progress variable PDF becomes bimodal, the variance reaches its maximum possible value of $\tilde{c}(1-\tilde{c})$. Conversely, in the limit of infinitely strong turbulence, the variance disappears due to perfect mixing. Accordingly, the blended, hybrid timescale model can be constructed as 


$$
\Omega_{\text {hybrid }}=(1-\eta) C_{\phi} \tau_{\text {turb }}{ }^{-1}+\eta \int_{0}^{1}\left\langle\chi_{c} \mid \zeta\right\rangle \widetilde{P}_{c}(\zeta) d \zeta / \widetilde{c^{\prime \prime 2}}
$$

The new timescale model, which is referred to as the hybrid model for the remainder of this work, is constructed to take advantage of the statistical data naturally available in a TPDF simulation and to properly describe the mixing rate in the flamelet limit. In the following section an a priori assessment of the accuracy of the new model is performed by comparison with DNS results.

\section{An a priori evaluation of the timescale model using DNS}

\subsection{DNS database description}

A 3-D DNS dataset of a lean $\mathrm{H}_{2}$-air turbulent premixed flame simulated with detailed chemistry [36] and mixture-averaged transport is utilized to evaluate the mixing timescale model. The flame configuration is described in detail in Ref. [36] and is briefly summarized here. In the simulation, two initially planar flames propagate into a slab of fresh reactants in the domain center as shown in Figure 1a. The temperature of the unburned reactants is $700 \mathrm{~K}$, the background pressure is atmospheric, and the equivalence ratio is $\phi=0.7$. The shear-driven flow sustains strong turbulence-flame interactions and is characteristic of practical conditions in gas turbines. The same DNS have been previously used in studies fractal flame characteristics [41] and in modeling in studies of different TPDF mixing models [27], one-dimensional turbulence models [42], and strained flamelet models [43].

The physical domain is $16 \mathrm{H} \times 20 \mathrm{H} \times 12 \mathrm{H}$ in the $\mathrm{x}, \mathrm{y}$, and $\mathrm{z}$ directions, respectively, where $\mathrm{H}$ is the initial fuel jet thickness. The size was selected to provide adequate statistical convergence and avoid scale-saturation over the simulation period. The boundaries in the streamwise (x) and spanwise (z) directions are periodic while non-reflecting outflow boundary conditions are applied in the transverse direction $(\mathrm{y})$. The simulation was performed using the Sandia DNS 
code, S3D [44]. The grid number of $\sim 7$ billion is selected to resolve the flame thickness with at least 14 grid points while the ratio of the Kolmogorov scale to grid size was 0.5 , following standard practice [45]. A detailed chemical kinetic model of hydrogen oxidation with 9 species and 21 reversible chemical reactions was employed [46]. Three cases are simulated in [36] by fixing the jet Reynolds number at $R e_{j e t}=U H / v_{0}=10,000$ and varying the jet Damköhler number, where $\mathrm{U}$ is the jet velocity and $v_{0}$ is the unburnt mixture viscosity. Each case is separated by a factor of approximately two in jet Damköhler number. From the three cases simulated, the two cases " $\mathrm{Da}^{-"}$ and $" \mathrm{Da}^{+"}$ are selected for the present study, which differ by a factor of four in Damköhler number. Relevant simulation parameters for each case are summarized in Table 1.

Table 1 - DNS Parameters

\begin{tabular}{c|c|c} 
& Case $\mathrm{Da}^{-}$ & ${\text {Case } \mathrm{Da}^{+}}^{+}$ \\
\hline $\mathrm{Da}_{\text {jet }}$ & 0.13 & 0.54 \\
$\mathrm{H}(\mathrm{mm})$ & 2.7 & 5.4 \\
$\mathrm{U}(\mathrm{m} / \mathrm{s})$ & 312.6 & 156.3 \\
$\Delta \mathrm{x}(\mu \mathrm{m})$ & 18 & 36 \\
$\Delta \mathrm{t}(\mathrm{ns})$ & 2.5 & 5 \\
$\mathrm{t}_{\mathrm{j}}=\mathrm{H} / \mathrm{U}(\mathrm{s})$ & $8.64 \mathrm{e}-6$ & $3.45 \mathrm{e}-5$ \\
\hline $\mathrm{S}_{\mathrm{L}}$ & \multicolumn{2}{|c}{$7.9 \mathrm{~m} / \mathrm{s}$} \\
$\delta_{\mathrm{L}}$ & \multicolumn{2}{|c}{$0.503 \mathrm{~mm}$}
\end{tabular}

Figure $1 \mathrm{~b}$ shows the trajectories on a regime diagram of the point in the domain that has the maximum turbulent Reynolds number, $R e_{t}=u^{\prime \prime} l_{t} / \tilde{v}$, at a given time where $u$ " is the RMS velocity fluctuation and $l_{t}$ is the integral length scale of the initial turbulence field, while $\tilde{v}$ is the mean viscosity. The trajectories are colored by the simulation time normalized by the characteristic jet time. The peak $\mathrm{Re}_{\mathrm{t}}$ for each case is approximately 1,000 and the Karlovitz number at the point of peak Reynolds number is 92 in Case $\mathrm{Da}^{-}$and 22 in Case $\mathrm{Da}^{+}$. The regime diagram shows that $\mathrm{Case} \mathrm{Da}^{-}$has $\mathrm{Da}$ smaller than unity throughout the simulation, while Case 

cases are in what is usually characterized as the thin reaction zones regime.

The DNS configuration allows for Favre-averaging in the streamwise and spanwise directions, as well as the use of symmetry across the domain centerline in the transverse direction. After averaging, the simulation is reduced to statistical dependence on the transverse direction and time. A reaction progress variable can be constructed based on the mass fraction of $\mathrm{H}_{2}$ as $c=\left(Y_{H 2}-Y_{H 2, u}\right) /\left(Y_{H 2, b}-Y_{H 2, u}\right)$, where the subscripts $\mathrm{u}$ and $\mathrm{b}$ refer to the unburnt and equilibrium states, respectively. A previous study on the performance of TPDF mixing models in this flame showed that differential diffusion can have a significant effect, and that using a progress variable definition based on the mass fraction of $\mathrm{H}_{2}$ provides accurate TPDF solutions [27]. Therefore, the progress variable definition based on $\mathrm{H}_{2}$ is used throughout the remainder of this work.

After Favre-averaging the progress variable field in the DNS, the mixing rate is computed as $\Omega=\widetilde{\chi_{c}} / \widetilde{c^{\prime \prime 2}}$ where the scalar dissipation rate is defined as $\widetilde{\chi_{c}}=2 \Gamma_{c} \nabla \widetilde{\nabla " \nabla c}$. The diffusivity of the progress variable, $\Gamma_{c}$, is defined as the molecular diffusivity of $\mathrm{H}_{2}$ [27]. The turbulent mixing rate is extracted from the DNS by computing the turbulent kinetic energy and its dissipation rate. The flamelet component of the hybrid timescale model is extracted by first computing the Favre-averaged PDF of the progress variable at each transverse location in space and at each time step. The conditional progress variable scalar dissipation rate is reconstructed from a freely propagating, 1D laminar flame calculated using PREMIX under the same unburnt conditions as the DNS. The product of the Favre PDF and the 1D conditional scalar dissipation rate are then integrated at each location in space and time to form the flamelet mixing rate in Equation 4. Finally, the hybrid timescale model, which blends together the turbulent and flamelet 
mixing rates using the segregation factor, is reconstructed from the DNS by computing the segregation factor at each location in space and time, and combining the turbulent mixing rate with the flamelet mixing rate according to Equation 5.

\subsection{A priori results and discussion}

The instantaneous temperature field for Case $\mathrm{Da}^{-}$is shown on a representative cross-section of the DNS domain at four important flame times in Figure 2. The two flames propagate inwards, and the $11^{\text {th }}$ jet time is approximately when the flame first begins to interact with the shear layer. At the $14^{\text {th }}$ jet time, the turbulence is considered to be fully developed, and the maximum flame wrinkling occurs near the $17^{\text {th }}$ jet time [36]. By the $20^{\text {th }}$ jet time, the flame is in its final stage and is burning the remainder of the fresh reactants at the domain center.

In the hybrid mixing rate model, the scalar dissipation requires closure while the scalar variance can be computed directly from the TPDF simulation. Therefore, we compare here the spatial and temporal variation of the progress variable scalar dissipation rate extracted from the DNS as well as the scalar dissipation rate implied by the new hybrid model, $\tilde{\chi}_{\text {hybrid }}=$ $\Omega_{\text {hybrid }} \widetilde{c^{\pi 2}}$, which are shown in the contour plots in Figure 3 . The values for both the DNS and modeled scalar dissipation rates are normalized for each DNS case by the maximum value across space and time of the DNS scalar dissipation rate, which is computed as: $\max \left(\tilde{\chi}_{c, D N S}(y, t)\right)$. The trajectory of the location in the flame brush with a mean progress variable of $\tilde{c}=0.5$ is overlaid on each plot, along with the trajectory of the leading edge $(\tilde{c}=0.01)$ and the trailing edge ( $\tilde{c}=0.95)$ of the flame brush. At the leading edge of the flame brush through the center of the flame, the hybrid timescale model accurately captures the spatial and temporal variation of the scalar dissipation rate. The peak magnitude of the scalar dissipation rate, which occurs in the 
center of the flame brush, is under-predicted by $18 \%$ for Case $\mathrm{Da}^{-}$and $10 \%$ for Case $\mathrm{Da}^{+}$. Towards the trailing edge of the flame brush the hybrid timescale model tends to under-predict the magnitude of the scalar dissipation rate for both cases. However, at this location in the flame brush, the mixing rate is less critical for accurate flame prediction as the burnt mixture is relaxing to chemical equilibrium.

The spatial and temporal distribution of the segregation factor, $\eta$, which blends together the contributions of the turbulent mixing and flamelet mixing components of the hybrid model is extracted from the DNS and shown in Figure 4. The segregation factor is larger in the more flamelet-like Case $\mathrm{Da}^{+}$than in Case $\mathrm{Da}^{-}$throughout space and time as expected. The variation of the segregation factor through the flame brush is shown in Figure 5. It can be seen that the segregation factor is small towards the leading edge of the flame in Case $\mathrm{Da}^{-}$, indicating the disruption of the preheat zone by the turbulence and the strong mixing in this region. Conversely, the segregation factor peaks towards the leading edge of the flame in the more flamelet-like Case $\mathrm{Da}^{+}$.

The scatter plots in Figure 6 and Figure 7 show the normalized averaged scalar dissipation rate variation through the flame brush for the DNS and the hybrid timescale model, along with each of the limiting components that are combined to form the hybrid timescale model for a random sample of locations across the entire spatial domain and time. As in Figure 3, the values for both the DNS and modeled scalar dissipation rates are normalized for each DNS case by the maximum value across space and time of the DNS scalar dissipation rate. Overlaid on the plots is the conditional average of the scalar dissipation rate, $\left\langle\widetilde{\chi}_{c} \mid \tilde{c}\right\rangle$, which is computed by conditionally averaging on the mean progress variable across the entire domain for all times. In both cases it can be seen that the passive scalar mixing rate approach of Equation 3 leads to a peak dissipation 
rate skewed more towards the burnt side of the flame brush, which is qualitatively different than the variation seen in the DNS data. For the standard mechanical-to-scalar timescale ratio value of 2.0, the turbulent mixing rate model under-predicts the mixing rate magnitude for both cases. Conversely, the flamelet model in Equation 4 tends to more accurately capture the variation through the flame brush of the dissipation rate, showing a peak on the unburnt side of the flame brush. However, the magnitude of the mixing rate tends to be over-predicted by the flamelet model, particularly for Case $\mathrm{Da}^{+}$. It can be seen from the scatter plots that the scalar dissipation rate extracted from the DNS lies in between the limiting values of the turbulent mixing rate and flamelet mixing rate throughout the flame brush.

The hybrid timescale model, which combines the limiting behavior of the turbulent mixing and flamelet models, accurately predicts the scalar dissipation rate magnitude and variation throughout most of the flame brush for both DNS cases as shown in Figure 8. The peak conditionally averaged mean scalar dissipation rate is under-predicted by $13 \%$ in Case $\mathrm{Da}^{-}$and $6 \%$ in Case $\mathrm{Da}^{+}$. It is notable that the hybrid timescale model tends to under-predict the dissipation rate in the post-flame zone. This is due to the value of the segregation factor in the post-flame zone, which skews the hybrid model more towards the passive scalar mixing behavior than the flamelet behavior in this location of the flame brush. However, as noted earlier, at this location in the flame brush most of the reaction has occurred and the mixture is relaxing to chemical equilibrium. Therefore accurate prediction of the mixing rate in the post-flame zone may not be of critical importance.

\section{A posteriori evaluation of new and existing timescale models}

We now perform an a posteriori TPDF evaluation of the new mixing rate model using the same DNS database from section 3 as a numerical test bed. The DNS database is used to supply 
the initial conditions and all time-varying inputs to the governing equations in the composition TPDF method, including the mean velocity, turbulent diffusion coefficient, and mixing rate model components. This approach, employed in previous studies $[9,47]$, deliberately removes uncertainties of turbulence and scalar transport modeling in order to expose only the effect of the mixing model. The same thermodynamic properties and chemical reaction mechanism used in the DNS are used in the TPDF simulations to limit the potential sources of modeling error to the closure of the molecular mixing term. For comparison purposes, we investigate the new mixing rate model coupled with the EMST micro-mixing model and compare it to the more popular mechanical timescale model as well as the mixing term closure proposed by Pope and Anand [34]. The EMST model was chosen because our previous study [47], which took the mixing timescale input directly from the DNS, demonstrated EMST's superiority over IEM and MC models for premixed flames. It is emphasized that the purpose of the present study is to evaluate the mixing timescale model, and not the mixing format, and thus it is possible that other untested mixing format models may produce similar results.

\subsection{TPDF Methodology}

The TPDF solver has been implemented in the RANS context using a hybrid particle-mesh methodology exactly as in several previous studies of mixing models $[9,27]$. For the given flame configuration, a 1D Eulerian mesh is generated covering half of the DNS domain in the transverse direction with a symmetric boundary condition accounting for the centerline. The Eulerian mesh is populated with notional Lagrangian particles, which obey the set of governing stochastic differential equations, Equations 6 and 7 [5,9], in physical and composition space:

$$
\begin{gathered}
d x^{*}(t)=\left[\widetilde{V}+\frac{\nabla \widetilde{\Gamma} \bar{\rho}}{\bar{\rho}}\right] d t+\left(2 \widetilde{\Gamma}_{t}\right)^{1 / 2} d W, \text { and } \\
d \phi^{*}(t)=[m] d t+\dot{\omega}\left(\phi^{*}\right) d t,
\end{gathered}
$$


where $x^{*}$ and $\boldsymbol{\phi}^{*}$ are the spatial location and composition, respectively, of a particle, $\tilde{V}$ is the mean transverse velocity, $\tilde{\Gamma}_{t}$ is the turbulent diffusion coefficient, $d W$ is the increment of a Wiener process, $\dot{\omega}\left(\boldsymbol{\phi}^{*}\right)$ is the chemical source term, and $[\mathrm{m}]$ is the rate of change in composition due to mixing. Note that mean drift by molecular diffusion is ignored in Equation 7 as it is found that the turbulent diffusion coefficient is larger than the molecular diffusion coefficient by at least an order of magnitude throughout the flame brush.

The mean transverse velocity, turbulent diffusivity, and mixing rate are all extracted directly from the DNS results at each time step and fed to the TPDF solver. The turbulent diffusion coefficient is computed as $\tilde{\Gamma}_{t}=\frac{|\widetilde{\rho v} c-\bar{\rho} \bar{v} \tilde{c}|}{\bar{\rho}|\nabla \tilde{c}|}$ where $v$ is the velocity, $\rho$ is the density, and $\mathrm{c}$ is the progress variable [2]. All input data required by the TPDF solver has been computed by Favreaveraging the DNS data in the statistically homogeneous $\mathrm{x}$ and $\mathrm{z}$ directions at each time step. The simulations are initialized from the DNS data at the $11^{\text {th }}$ jet time, which is when the flame begins to interact with the shear layer.

In the current study, the stochastic governing equation for the composition vector, Equation 7, can evolve over time in two ways. In the first method, the EMST mixing model is used to close the molecular mixing term and is supplied with either the turbulent mixing rate or the new hybrid model mixing rate, which have been extracted from the DNS as described in section 3. The chemical source term is integrated using a 6-stage, 4th order Runge-Kutta numerical scheme [48].

In the second method, the laminar flamelet closure of Pope and Anand [34] is used to advance the particle composition. In this method, the mean progress variable is first computed for each cell. In cells near the leading $(\tilde{c}<0.05)$ and trailing $(\tilde{c}>0.95)$ edges of the flame 
brush where turbulent mixing is expected to be the dominant generator of scalar gradients, the particle composition is advanced in the same manner as in the first method using the turbulent mixing rate. For all other cells, the progress variable of each particle is calculated and the evolution of the composition vector is then computed according to Equation 8, where the diffusion and reaction contributions are interpolated from the 1D laminar flame solution for the given value of the particle progress variable.

$$
\mathrm{d} \phi^{*}(\mathrm{t})=[\nabla \Gamma \nabla \phi+\dot{\omega}(\phi)]_{\mathrm{c}=\mathrm{c}^{*}} \mathrm{dt}
$$

In this approach, particles in the middle of the flame brush evolve as if they were embedded in a locally 1D laminar flamelet. This laminar flamelet closure method is referred to as Pope's method through the remainder of this work.

Based on the convergence study performed in [27], all results in this paper for Case $\mathrm{Da}^{-}$ are generated using 300 cells, 4,000 particles per cell, and a time step of $5 \mathrm{~ns}$. All results for Case $\mathrm{Da}^{+}$are generated using 600 cells, 4,000 particles per cell, and a time step of $10 \mathrm{~ns}$. It is noted that the TPDF solver used in this study does not perform any smoothing, and thus the convergence rate is slow and requires significantly more particles. If smoothing were to be applied, the convergence rate would be much faster, and a particle level of 20-100 particles per cell, as found elsewhere in the literature, could be expected to achieve a converged solution [49].

\subsection{Results and Discussion}

The predictions of the TPDF solution with the three closure methods described above are compared to the DNS data at three important time instances in the flame evolution. In the figures that follow, the results denoted by "Pope" refer to the solution computed using Pope and Anand's laminar flamelet closure; the results denoted by "Turb. Mixing" refer to the solution computed using the EMST mixing model and the turbulent mixing timescale in Equation 3; and 
the results denoted by "Hybrid" refer to the solution computed using the EMST mixing model and the hybrid mixing timescale in Equation 5. The TPDF mean and RMS temperature distribution are compared to the DNS data for both cases in Figure 9. All three methods provide reasonably accurate predictions of the mean spatial temperature profile in Case $\mathrm{Da}^{-}$. Pope's method provides a poor approximation to the RMS temperature field as the decay of scalar variance because turbulence is not properly described in this closure in the present high $\mathrm{Ka}$ conditions. In Case $\mathrm{Da}^{+}$, Pope's closure performs poorly for both the mean and RMS temperature fields, simply propagating the flame elements along as if it were laminar and not capturing the turbulent flame acceleration found in this configuration. The relative success of Pope's closure for Case $\mathrm{Da}^{-}$and failure for Case $\mathrm{Da}^{+}$can be attributed to the influence of the EMST mixing model, which is invoked at the leading edge of the flame brush. While the timescales implied by the right-hand side of Equation 8 and that of the hybrid mixing rate model are similar in magnitude, the use of the EMST model coupled with the turbulent mixing rate at the leading edge of the flame controls the flame propagation in Pope's model until the flame brush begins to interact with the symmetry boundary. The performance of Pope's model is dependent on the specification of the value of $\tilde{c}$ at which the transition between the use of the EMST mixing model and the laminar flamelet closure occurs. Note that this transition is needed as the laminar flamelet closure is ill-conditioned as the progress variable tends towards zero. For the flames considered here, better agreement may be obtained by tuning the $\tilde{c}$ value at which the transition occurs, however the optimal value for transition is case-dependent. The turbulent mixing rate provides a reasonable solution in Case $\mathrm{Da}^{-}$and therefore so does Pope's closure, while both fail for Case $\mathrm{Da}^{+}$. 
The EMST mixing model coupled with the turbulent mixing rate model shows acceptable accuracy for the mean temperature behavior in Case $\mathrm{Da}^{-}$, but provides increasingly inaccurate predictions of the RMS temperature as the flame progresses. For Case $\mathrm{Da}^{+}$, the turbulent mixing rate under-predicts the flame propagation and is entirely inaccurate for both the mean and RMS profiles. While the mixing rate constant, $C_{\phi}$, can in principle be tuned to achieve closer agreement for the mean temperature field predictions, previous studies have shown that even for a flame in the same configuration, the optimal choice of $C_{\phi}$ can vary by a factor of two or more [27], which would be problematic for practical application where $C_{\phi}$ is not known a priori. It is clear from the results that the turbulent mixing rate alone does not have the appropriate spatial and temporal distribution to represent the scalar mixing in this premixed flame.

The TPDF solution using the hybrid mixing rate model shows excellent agreement with the DNS mean and RMS temperature fields for both cases. As shown in section 3, the hybrid mixing rate model is able to accurately capture the spatial and temporal distribution of the mixing rate, particularly in the preheat zone. This leads to close agreement of the TPDF solution with the DNS both in space and time without the need to arbitrarily choose the mixing rate constant $C_{\phi}$ to achieve an accurate prediction.

To evaluate the influence of the closure models on flame propagation, the turbulent flame speed, $\mathrm{S}_{\mathrm{T}}$, defined based on the global consumption rate is constructed as:

$$
\mathrm{S}_{\mathrm{T}}=\frac{1}{\rho_{\mathrm{u}}} \int_{0}^{\mathrm{ymax}} \overline{\dot{\omega}_{\mathrm{c}}}(\mathrm{y}) \mathrm{dy}
$$

where $\overline{\dot{\omega}_{c}}$ is the mean mass production rate of the progress variable and $\rho_{u}$ is the density in the unburnt reactants. The turbulent flame speed normalized by the laminar flame speed is shown in Figure 10. The hybrid timescale model closure provides the best prediction of the temporal flame 
speed variation for both DNS cases. The turbulent mixing rate model provides a reasonable approximation for Case $\mathrm{Da}^{-}$, but under-predicts the flame speed for Case $\mathrm{Da}^{+}$. When evaluated by the global consumption speed, Pope's closure fails for both cases as the flame acceleration is not properly captured.

To more closely examine the behavior of all three closures, the major species spatial profile predictions are shown at the $17^{\text {th }}$ jet time in Figure 11 and Figure 12. The major species profiles behave similarly to the temperature field, with all three models providing reasonable predictions of the mean fields for Case $\mathrm{Da}^{-}$. However, only the hybrid mixing rate model is able to accurately capture the correct flame structure in Case $\mathrm{Da}^{+}$.

Similarly, the minor species spatial profiles are shown at the $17^{\text {th }}$ jet time in Figure 13 and Figure 14. It can be seen that the solution produced by Pope's closure is less accurate for the minor species profiles than for the major species in Case $\mathrm{Da}^{-}$. This can be attributed to the fact that turbulence-chemistry interaction effects are not accurately accounted for in the middle of the flame brush in this approach. The turbulent mixing rate and hybrid mixing rate model show similar levels of accuracy for the minor species as for the major species in Case $\mathrm{Da}^{-}$. As with the major species predictions in Case $\mathrm{Da}^{+}$, only the hybrid mixing rate model is able to accurately capture the flame structure. The hybrid mixing rate model shows close agreement with the DNS data for all three minor species plotted.

\section{Summary and Conclusions}

In this work, a new hybrid mixing rate model is developed which aims to account for the scalar mixing rate behavior in both the turbulent mixing limit and the flamelet limit. In the turbulent mixing limit, the model uses the classical expression assuming the rate of scalar mixing is inversely proportional to the turbulence timescale. In the flamelet limit, the new timescale 
model reconstructs the mixing rate by integrating the local PDF and the conditional scalar dissipation rate from a 1-D freely propagating premixed flame. The two mixing timescale model components are then blended together using the segregation factor to more accurately describe the local mixing behavior. The new hybrid timescale model is notable for its treatment of the flamelet mixing limit, an area where existing timescale models do not properly recover the correct mixing behavior [33].

Using DNS data of a temporally evolving premixed hydrogen-air slot jet flame, an a priori comparison of the new mixing rate model is performed. The a priori evaluation, which reconstructs each component of the hybrid model including the segregation factor from the DNS, demonstrates that the commonly used turbulent mixing rate model does not have the correct spatial or temporal distribution for this flame. Additionally, the commonly chosen mechanicalto-scalar timescale ratio of 2.0 is found to largely under-predict the mixing rate magnitude throughout the flame brush. Conversely, the new hybrid timescale model more accurately captures the scalar mixing rate spatial profiles without the need for an arbitrarily chosen timescale constant.

Finally, an a postereori assessment using DNS as a numerical test bed for the TPDF solution is performed, comparing the new mixing rate model coupled with the EMST mixing model to the commonly used turbulent mixing rate model and the laminar flamelet closure of Pope and Anand [23]. The TPDF solution demonstrates that of the three closures tested in this work, the hybrid mixing rate model coupled with the EMST mixing model provides the best accuracy for this flame. The new model is able to accurately capture the flame structure in both cases tested, whereas the turbulent mixing rate model and the laminar flamelet closure under-predict the flame propagation. 


\section{Acknowledgements}

The work at the University of Connecticut was supported by the Chemical Sciences, Geosciences and Biosciences Division, Office of Basic Energy Sciences, Office of Science, U.S. Department of Energy, under Grant DE-SC0008622. The work at Tsinghua University was supported by National Natural Science Foundation of China 91441202 and 51476087. The work at UNSW was supported by the Australian Research Council.

\section{References}

[1] S.B. Pope, PDF methods for turbulent reactive flows, Prog. Energy Combust. Sci. 11 (1985) 119-192.

[2] L. Valiño, A Field Monte Carlo Formulation for Calculating the Probability Density Function of a Single Scalar in a Turbulent Flow, Flow, Turb. Combust. 60 (1998) 157-172.

[3] E.E. O'Brien, The probability density function (pdf) approach to reacting turbulent flows, in: P.A. Libby, F.A. Williams (Eds.), Turbulent Reacting Flows, Springer Berlin Heidelberg, Berlin, Heidelberg, 1980, pp. 185-218.

[4] Libby, Paul A., Williams,F.A., Turbulent Reacting Flows, Academic Press, London; New York, 1994.

[5] D.C. Haworth, Progress in probability density function methods for turbulent reacting flows, Prog. Energy Combust. Sci. 36 (2010) 168-259.

[6] R.C. Flagan, J.P. Appleton, A stochastic model of turbulent mixing with chemical reaction: Nitric oxide formation in a plug-flow burner, Combust. Flame. 23 (1974) 249-267.

[7] R.P. Lindstedt, E.M. Vaos, Transported PDF modeling of high-Reynolds-number premixed turbulent flames, Combust. Flame. 145 (2006) 495-511.

[8] M. Stöllinger, S. Heinz, Evaluation of scalar mixing and time scale models in PDF simulations of a turbulent premixed flame, Combust. Flame. 157 (2010) 1671-1685.

[9] A. Krisman, J.C.K. Tang, E.R. Hawkes, D.O. Lignell, J.H. Chen, A DNS evaluation of mixing models for transported PDF modelling of turbulent nonpremixed flames, Combust. Flame. (2014) 1-22.

[10] D.H. Rowinski, S.B. Pope, Computational study of lean premixed turbulent flames using RANS/PDF and LES/PDF methods, Combust. Theory Mod. 17 (2013) 610-656.

[11] D.H. Rowinski, S.B. Pope, PDF calculations of piloted premixed jet flames, Combust. Theory Mod. 15 (2011) 245-266. 
[12] S. Mitarai, J.J. Riley, G. Kosály, Testing of mixing models for Monte Carlo probability density function simulations, Phys. Fluids. 17 (2005) 047101.

[13] R. Lindstedt, E. Vaos, Modeling of mixing processes in non-isothermal and combusting flows, in: C. Dopazo (ed.), Advances in Turbulence, Vol. 8, 493-496.

[14] M.J. Dunn, A.R. Masri, R.W. Bilger, A new piloted premixed jet burner to study strong finite-rate chemistry effects, Combust. Flame. 151 (2007) 46-60.

[15] J.C.D. J. Villermaux, Proc. 2nd Int. Symp. on Chem. Reaction Eng. (1972) 1-13.

[16] C. Dopazo, E.E. O'Brien, An approach to the autoignition of a turbulent mixture, Acta Astronaut. 1 (1974) 1239-1266.

[17] R.L. Curl, Dispersed phase mixing: I. Theory and effects in simple reactors, AIChE J. 9 (1963) 175-181.

[18] J. Janicka, W. Kolbe, W. Kollmann, Closure of the transport equation for the probability density function of turbulent scalar fields, J. Non Equilib. Thermodyn. 4 (1979) 47-66.

[19] C. Dopazo, Relaxation of initial probability density functions in the turbulent convection of scalar fields, Phys. Fluids. 22 (1979) 20-30.

[20] S. Subramaniam, S.B. Pope, A mixing model for turbulent reactive flows based on Euclidean minimum spanning trees, Combust. Flame. 115 (1998) 487-514.

[21] Z. Ren, S.B. Pope, An investigation of the performance of turbulent mixing models, Combust. Flame. 136 (2004) 208-216.

[22] L. Valiño, C. Dopazo, A binomial Langevin model for turbulent mixing, Phys. Fluids A. 3 (1991) 3034-3037.

[23] H. Chen, S. Chen, R.H. Kraichnan, Probability distribution of a stochastically advected scalar field, Phys. Rev. Lett. 63 (1989) 2657-2660.

[24] S.B. Pope, Mapping closures for turbulent mixing and reaction, Theor. Comput. Fluid Dyn. 2 (1991) 255-270.

[25] S. Subramaniam, S.B. Pope, Comparison of mixing model performance for nonpremixed turbulent reactive flow, Combust. Flame. 117 (1999) 732-754.

[26] B.R.O. Fox, C.M. Cha, P. Trouillet, Lagrangian PDF mixing models for reacting flows, (2002). 
[27] M. Kuron, Z. Ren, E. Hawkes, H. Zhou, J. Tang, J.H. Chen, T. Lu, Performance of transported PDF mixing models in a turbulent premixed flame, Proceedings of the Combustion Institute. (2016) in press.

[28] D.H. Rowinski, S.B. Pope, An investigation of mixing in a three-stream turbulent jet, Phys. Fluids. 25 (2013) 105105-105105.

[29] N. Swaminathan, K. Bray, Effect of dilatation on scalar dissipation in turbulent premixed flames, Combust. Flame. 143 (2005) 549-565.

[30] H. Kolla, Scalar Dissipation Rate based Flamelet Modelling of Turbulent Premixed Flames, (2009).

[31] H. Kolla, J.W. Rogerson, N. Chakraborty, N. Swaminathan, Scalar Dissipation Rate Modeling and its Validation, Combust. Sci. Technol. 181 (2009) 518-535.

[32] A. Mura, R. Borghi, Towards an extended scalar dissipation equation for turbulent premixed combustion, Combust. Flame. 133 (2003) 193-196.

[33] K. Bray, M. Champion, P.a. Libby, N. Swaminathan, Scalar dissipation and mean reaction rates in premixed turbulent combustion, Combust. Flame. 158 (2011) 2017-2022.

[34] S.B. Pope, M.S. Anand, Flamelet and distributed combustion in premixed turbulent flames, Proc. Combust. Inst. 20 (1984) 403-410.

[35] Z. Zhou, S. Li, H. Wang, Z. Ren, An Investigation of a Hybrid Mixing Model for PDF Simulations of Turbulent Premixed Flames, 68th Annual Meeting of the APS Division of Fluid Dynamics. (2015).

[36] E.R. Hawkes, O. Chatakonda, H. Kolla, A.R. Kerstein, J.H. Chen, A petascale direct numerical simulation study of the modelling of flame wrinkling for large-eddy simulations in intense turbulence, Combust. Flame. 159 (2012) 2690-2703.

[37] T. Mantel, R. Borghi, A new model of premixed wrinkled flame propagation based on a scalar dissipation equation, Combust. Flame. 96 (1994) 443-457.

[38] C. Béguier, I. Dekeyser, B.E. Launder, Ratio of scalar and velocity dissipation time scales in shear flow turbulence, Phys. Fluids. 21 (1978) 307-310.

[39] E.R. Hawkes, R. Sankaran, J.C. Sutherland, J.H. Chen, Scalar mixing in direct numerical simulations of temporally evolving plane jet flames with skeletal $\mathrm{CO} / \mathrm{H} 2$ kinetics, Proc. Combust. Inst. 31 (2007) 1633-1640.

[40] N. Swaminathan, R.W. Bilger, Scalar dissipation, diffusion and dilatation in turbulent H-2air premixed flames with complex chemistry, Combust. Theory Mod. 5 (2001) 429-446. 
[41] O. Chatakonda, E.R. Hawkes, A.J. Aspden, A.R. Kerstein, H. Kolla, J.H. Chen, On the fractal characteristics of low Damköhler number flames, Combust. Flame. 160 (2013) 2422-2433.

[42] N. Punati, H. Wang, E.R. Hawkes, J.C. Sutherland, One-Dimensional Modeling of Turbulent Premixed Jet Flames - Comparison to DNS, Flow, Turb. Combust. (2016).

[43] P. Trisjono, K. Kleinheinz, E.R. Hawkes, H. Pitsch, Modeling turbulence-chemistry interaction in lean premixed hydrogen flames with a strained flamelet model, Combust. Flame. Submitted (2015).

[44] J.H. Chen, A. Choudhary, B. de Supinski, M. DeVries, E.R. Hawkes, S. Klasky, W.K. Liao, K.L. Ma, J. Mellor-Crummey, N. Podhorszki, R. Sankaran, S. Shende, C.S. Yoo, Terascale direct numerical simulations of turbulent combustion using S3D, Comp. Sci. Disc. 2 (2009) 015001-015001.

[45] S.B. Pope, Turbulent Flows, Cambridge University Press, 2000.

[46] J. Li, Z. Zhao, A. Kazakov, F.L. Dryer, An updated comprehensive kinetic model of hydrogen combustion, Int J Chem Kinet. 36 (2004) 566-575.

[47] M. Kuron, Z. Ren, H. Kolla, E.R. Hawkes, J.H. Chen, T. Lu, An Investigation of the Scalar Dissipation Rate Behavior in a Premixed Hydrogen Flame 9th U. S. National Combustion Meeting.

[48] C.A. Kennedy, M.H. Carpenter, R.M. Lewis, Low-storage, explicit Runge-Kutta schemes for the compressible Navier-Stokes equations, Appl. Numer. Math. 35 (2000) 177-219.

[49] S. Viswanathan, H. Wang, S.B. Pope, Numerical implementation of mixing and molecular transport in LES/PDF studies of turbulent reacting flows, J. Comp. Phys. 230 (2011) 6916-6957. 
(a)

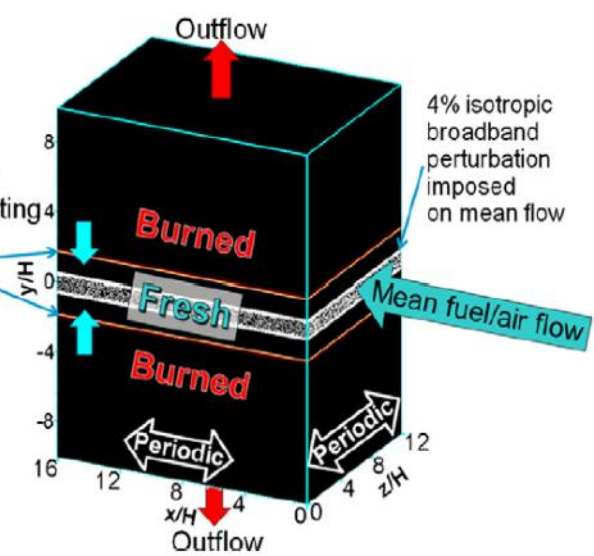

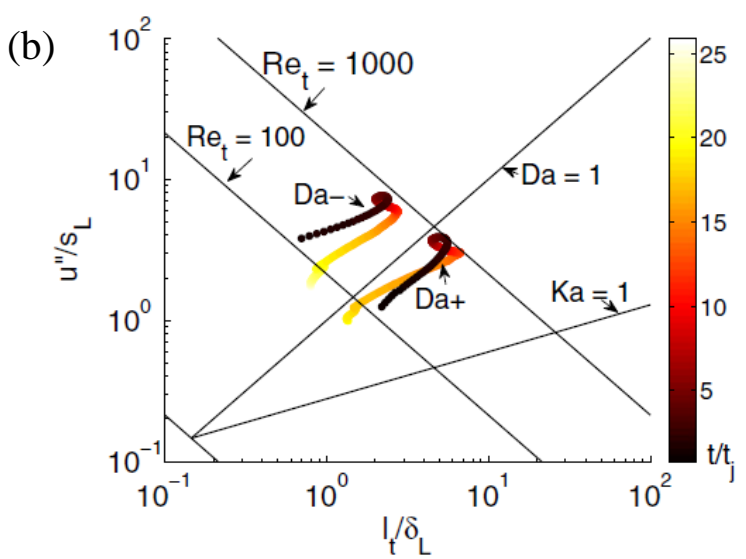

Figure 1 - (a) Flame configuration and (b) regime diagram of the DNS data. The temporal evolution of the $\mathrm{Da}^{+}$and $\mathrm{Da}^{-}$cases is overlaid on the diagram. Figures are reproduced from [36].
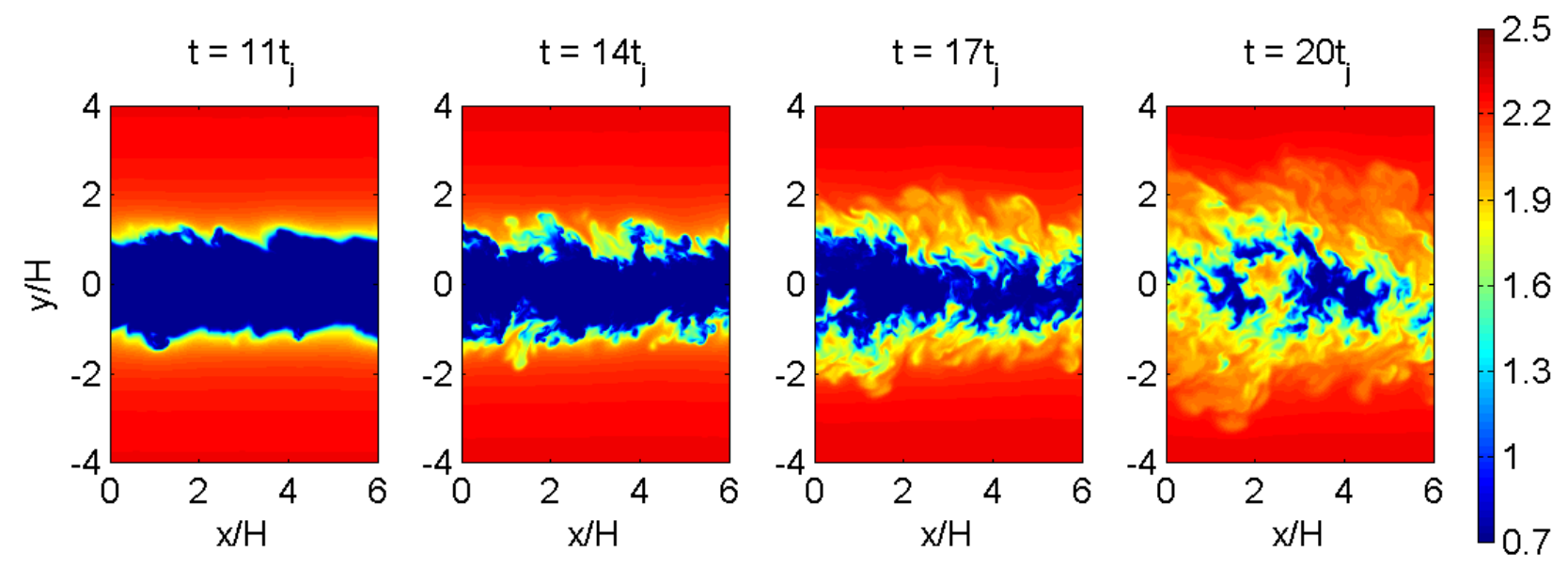

Figure 2 - Instantaneous temperature (T/1000 K) field on a representative section of the central spanwise plane at several time instances from the DNS for Case $\mathrm{Da}^{-}$. 


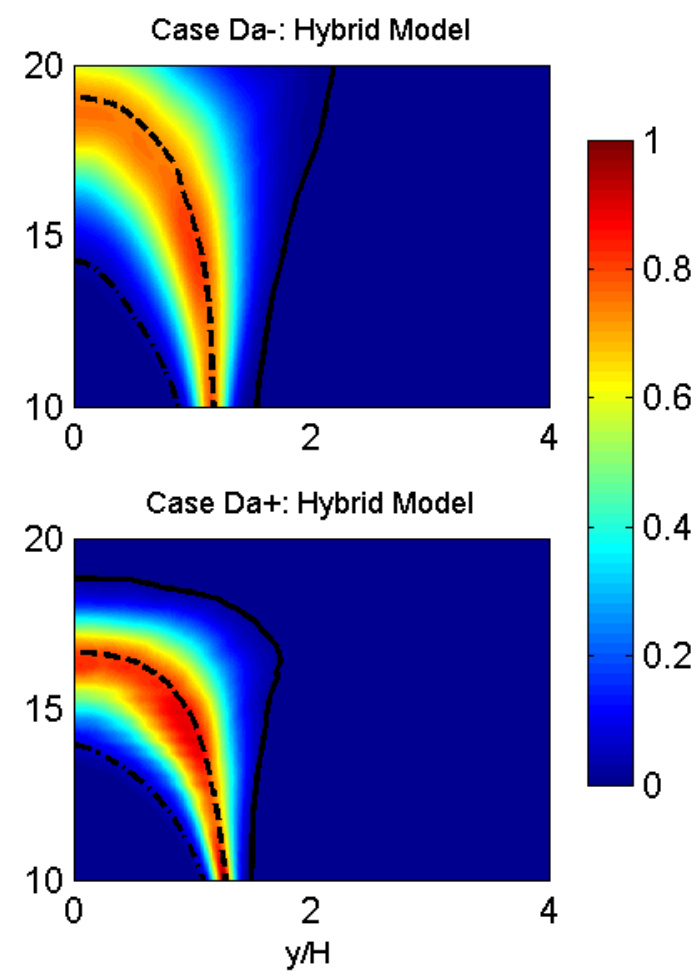

Figure 3 - Scalar dissipation rate normalized by the maximum DNS value for Case $\mathrm{Da}^{-}$(top) and Case $\mathrm{Da}^{+}$(bottom). Left Column: DNS. Right Column: Hybrid model from Eqn. (5). Dash-dotted line: $\tilde{c}=0.01$, dashed line: $\tilde{c}=0.5$, solid line: $\tilde{c}=0.95$.
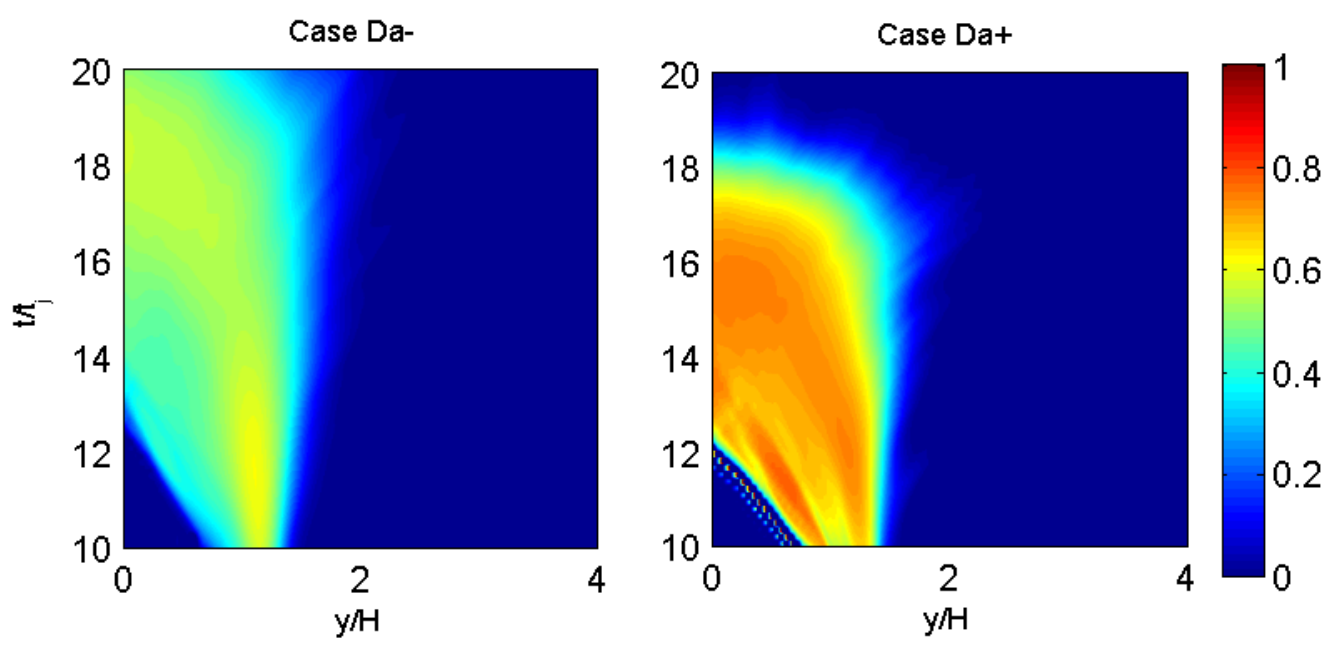

Figure 4 - The segregation factor $\eta$ reconstructed from the DNS data. 


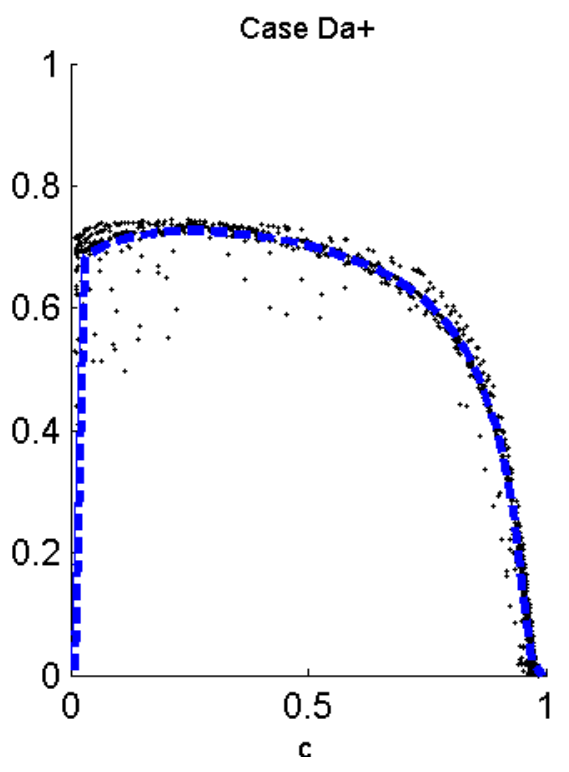

Figure 5 - The segregation factor $\eta$ vs. mean progress variable. Dashed blue line represents the segregation factor conditionally averaged on the mean progress variable.
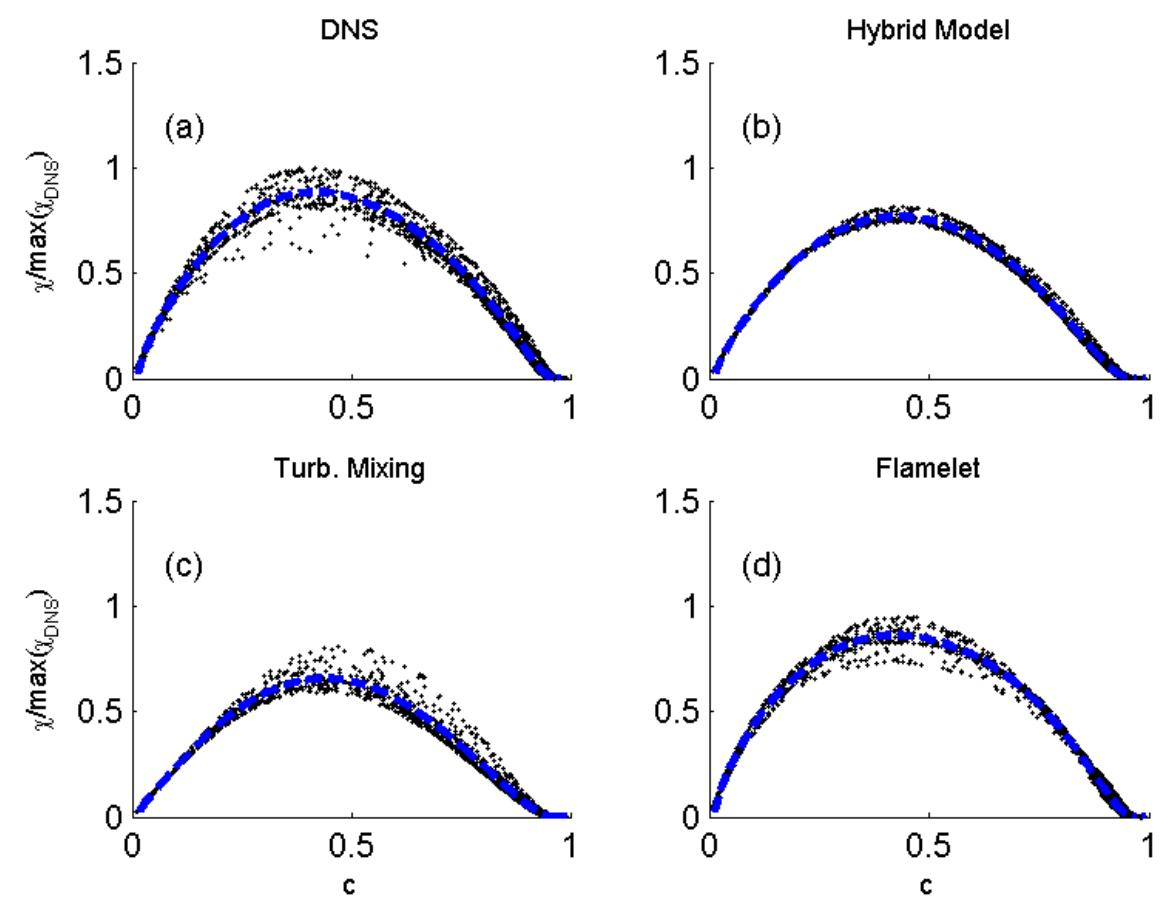

Figure 6 - Scalar dissipation rate normalized by the maximum DNS value vs. mean progress variable for Case Da'. (a) DNS. (b) Equation 5. (c) Equation 3. (d) Equation 4. Dashed black line represents the mean scalar dissipation rate conditionally averaged on the mean progress variable. 

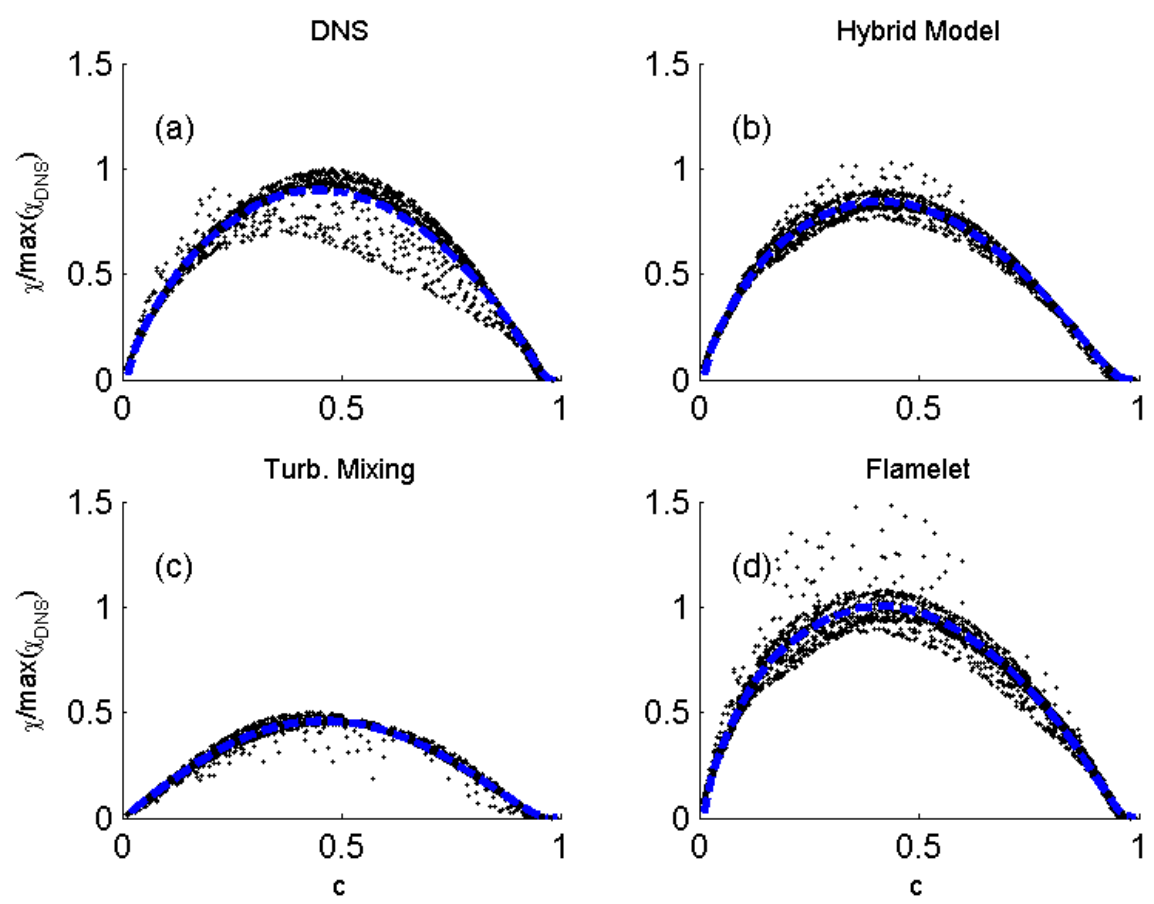

Figure 7 - Scalar dissipation rate normalized by the maximum DNS value vs. mean progress variable for Case $\mathrm{Da}^{+}$. (a) DNS. (b) Equation 5. (c) Equation 3. (d) Equation 4. Dashed black line represents the mean scalar dissipation rate conditionally averaged on the mean progress variable.
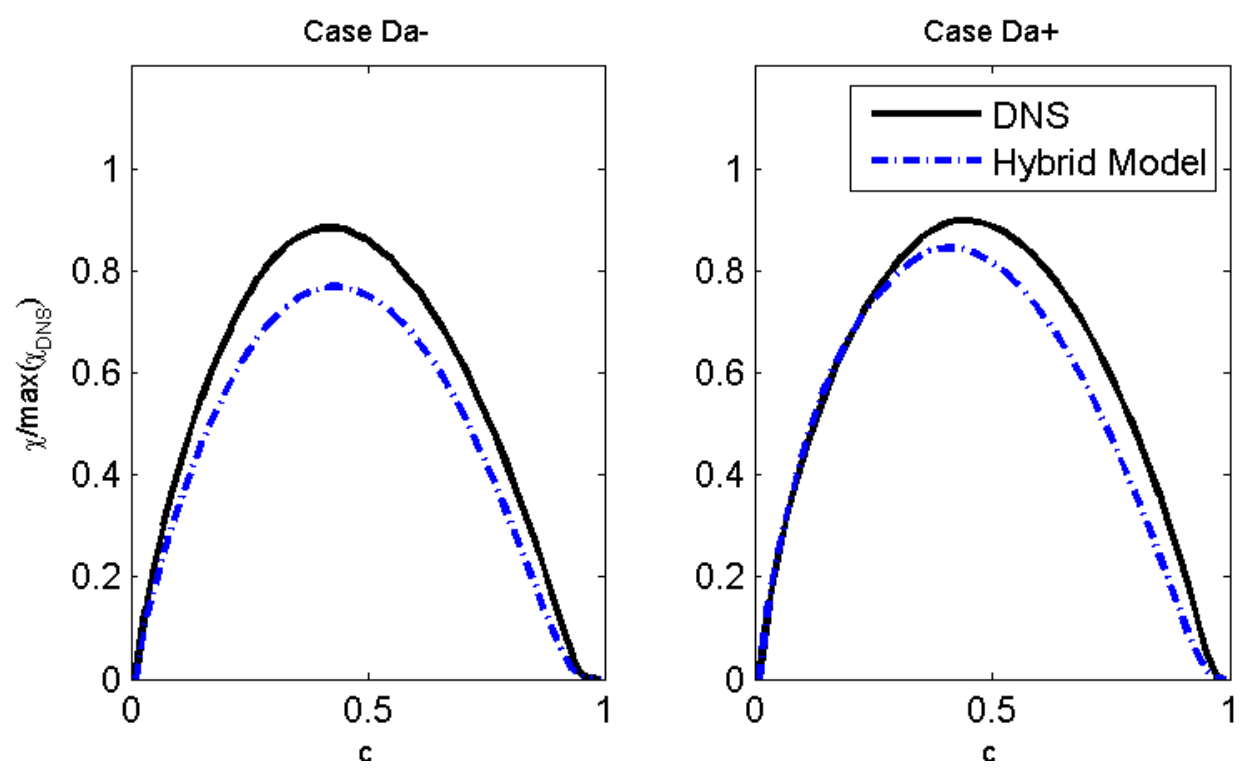

Figure 8 - Normalized mean scalar dissipation rate conditionally averaged on the mean progress variable. 

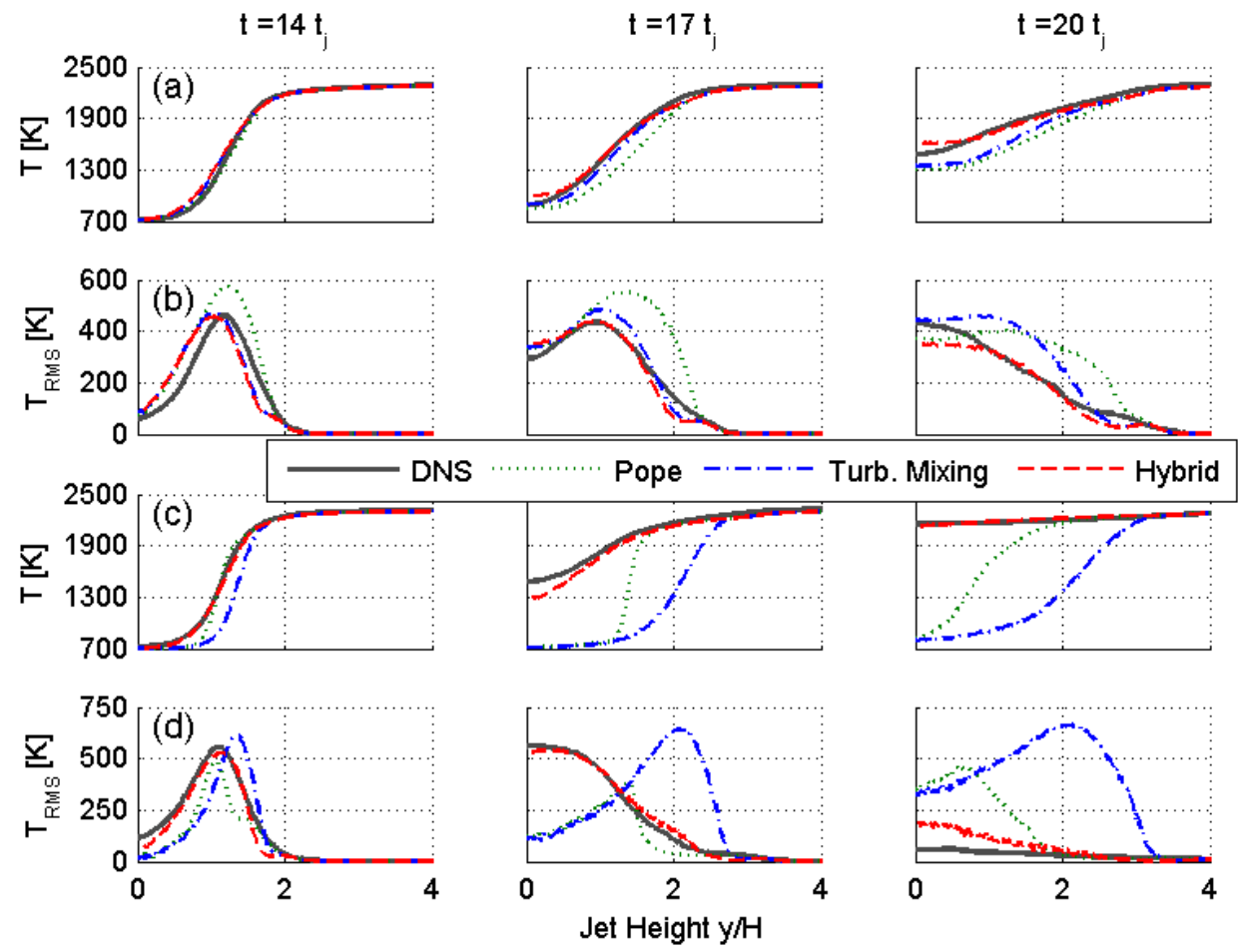

Figure 9 - Predicted mean and RMS spatial temperature profiles from the DNS and TPDF solutions. Rows (a) and (b): Case $\mathrm{Da}^{-}$. Rows (c) and (d): Case $\mathrm{Da}^{+}$.

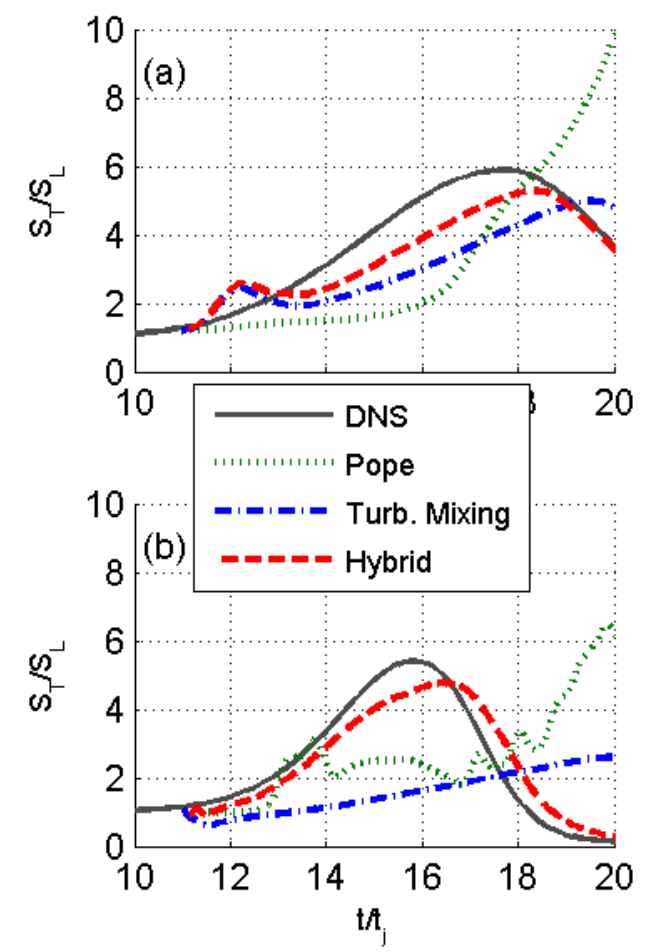

Figure 10 - Turbulent flame speed vs. time. (a) Case $\mathrm{Da}^{-}$. (b) Case $\mathrm{Da}^{+}$ 

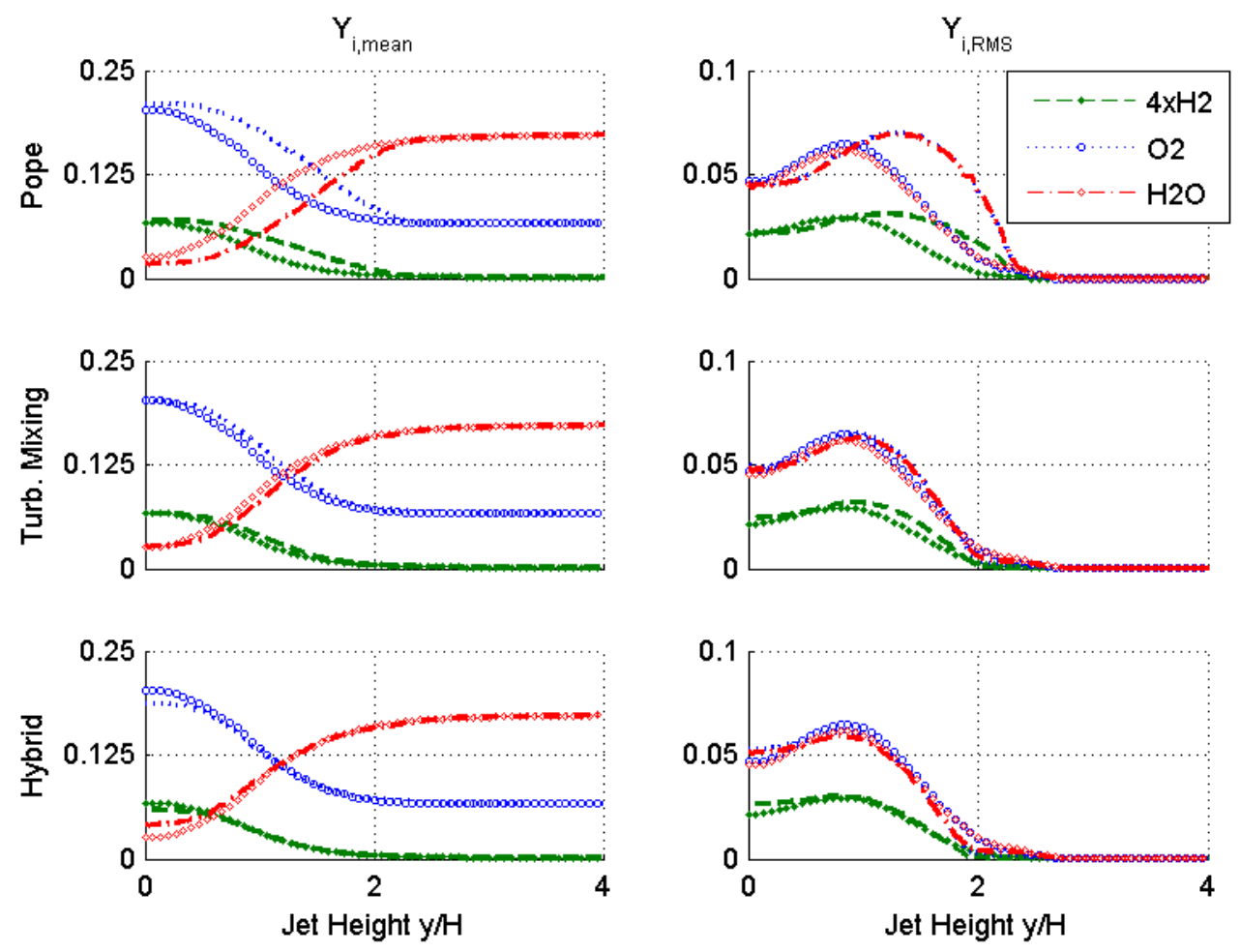

Figure 11 - Major species mean and RMS profiles at $17 \mathrm{t}_{\mathrm{j}}$ for case Da'. Markers: DNS. Lines: TPDF.
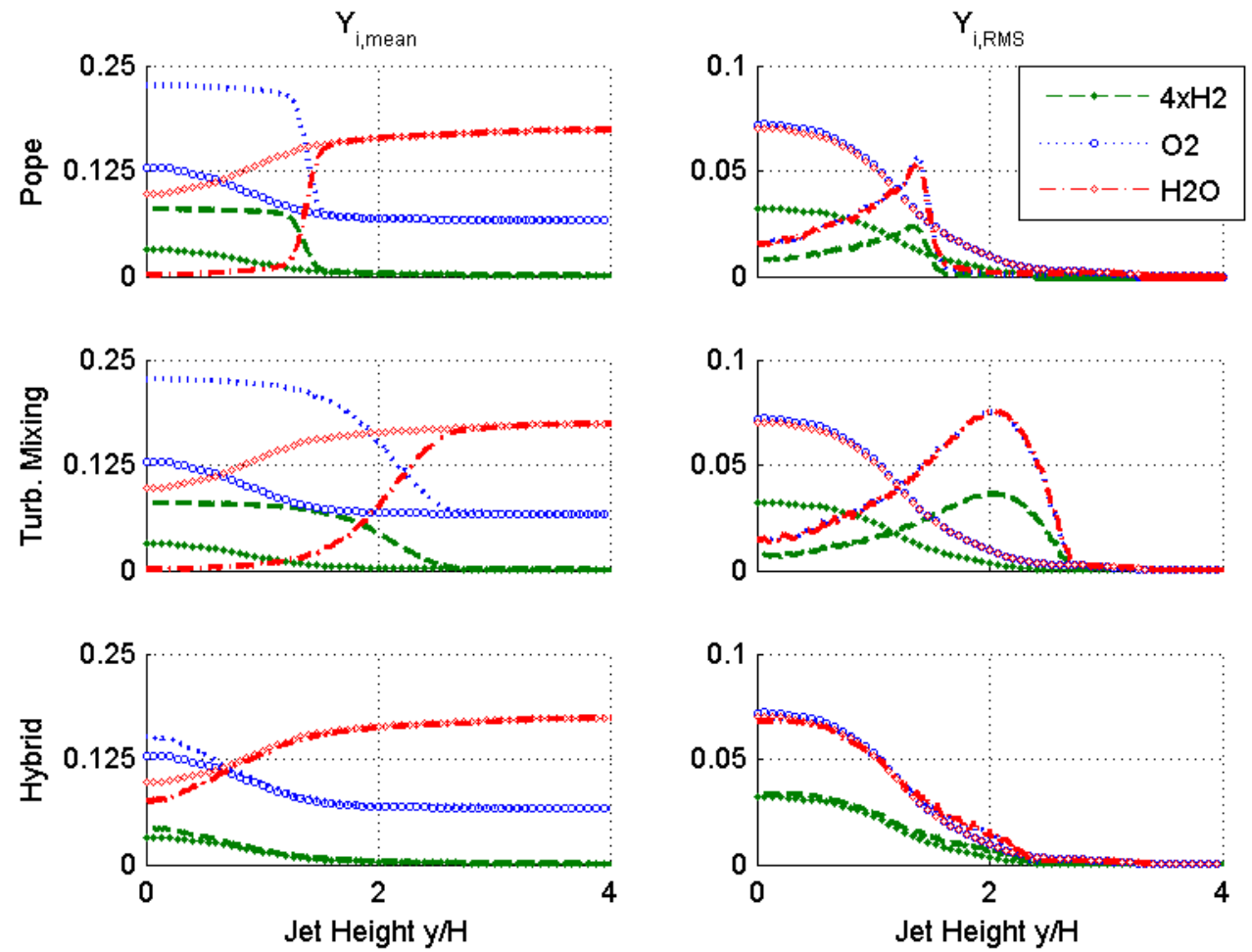

Figure 12 - Major species mean and RMS profiles at $17 \mathrm{t}_{\mathrm{j}}$ for case $\mathrm{Da}^{+}$. Markers: DNS. Lines: TPDF. 

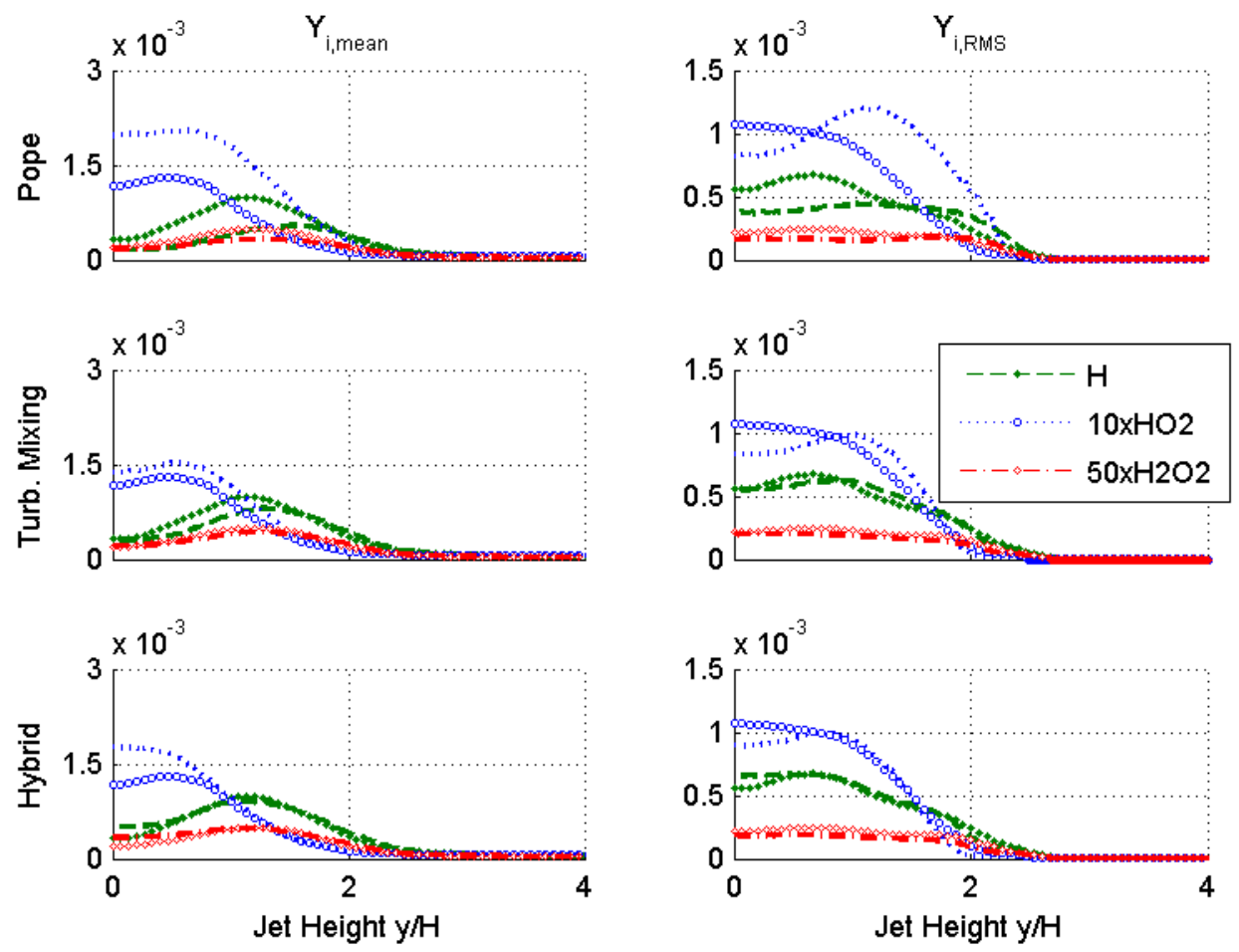

Figure 13 - Selected minor species mean and RMS profiles at $17 \mathrm{t}_{\mathrm{j}}$ for case $\mathrm{Da}$. Markers: DNS. Lines: TPDF.
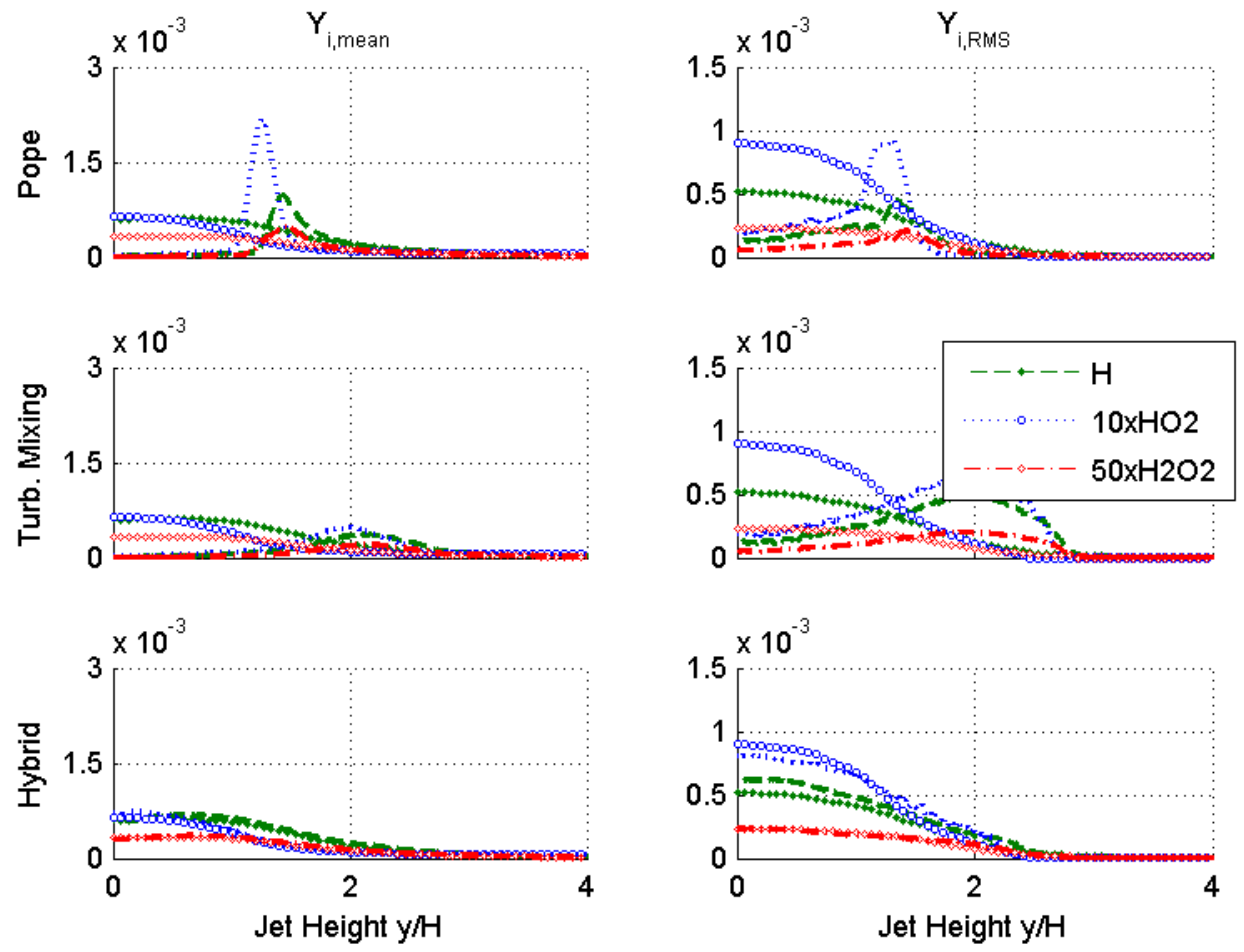

Figure 14 - Selected minor species mean and RMS profiles at $17 \mathrm{t}_{\mathrm{j}}$ for case Da- Markers: DNS. Lines: TPDF. 